\title{
Effects of Different Drying Methods on Antioxidant Activity of Polysaccharides from Antler Mushroom
}

\author{
Bing Xu, Yi Dai, Shiyu Xu, Jianjun Liu, Botao Hu, Xiao Ding, Xinyi Wu and *Yiyong Chen \\ School of Biology and Food Engineering, Changshu Institute of Technology, Changshu 215500, China
}

\author{
Article history \\ Received: 28-04-2021 \\ Revised: $10-06-2021$ \\ Accepted: 23-06-2021 \\ Corresponding Author: \\ Yiyong Chen \\ School of Biology and Food \\ Engineering, Changshu \\ Institute of Technology, \\ Changshu 215500, China \\ Email: greenpop6688@126.com
}

\begin{abstract}
In this study, three polysaccharides (AMP-H, AMP-V and AMPF) were obtained from polysaccharides of Antler Mushroom (AMP) by hot air drying, vacuum drying and freeze drying, respectively. The chemical composition and antioxidant capacity of AMP-H, AMP-V and AMP-F were investigated. The results showed that the AMP was an acidic polysaccharide containing a small amount of protein. The drying method did not affect the monosaccharide type, but changed the composition ratio of monosaccharide and the content of uronic acid. The content of uronic acid in AMP-H was lower significantly than that in AMP-V and AMP-F $(p<0.05)$. Antioxidant studies showed that AMP-H, AMP-V and AMP-F had strong scavenging ability against hydroxyl radical, superoxide anion radical and DPPH radical. Within a certain concentration range, antioxidant capacity of AMP-H, AMP$\mathrm{V}$ and AMP-F was positively correlated with the mass concentration. The drying method had different effects on the antioxidant capacity of AMP. Compared with AMP-H and AMP-V, AMP-F had the strongest antioxidant activity. Based on the above results, freeze drying is the best drying method suitable for AMP preparation among the three tested methods.
\end{abstract}

Keywords: Antler Mushroom Polysaccharide, Drying Mode, Chemical Composition, Antioxidant

\section{Introduction}

Velvet Antler mushroom (Lyophyllum decastes), is a kind of rare edible mushroom with medicinal properties. It belongs to order Agaricales, dried mushroom family and Lyophyllum (Arana-Gabriel et al., 2018). The name Antler mushroom is derived from mushroom shape that resembles a broom, coral and a young antler. Antler mushroom is rich in nutrients, including protein, vitamins, dietary fiber, eight essential amino acids, vitamins and polysaccharides. $\beta$-dextran is main functional ingredients of polysaccharides in Antler mushroom (AMP) containing $\beta-1,3-\mathrm{D}$ dextran and $\beta-1,6-\mathrm{D}$ dextran (Zheng et al., 2013). Studies have shown that AMP has multiple effects (Lee et al., 2011). It is a low-calorie and low-fat health product that can lower blood pressure and cholesterol and prevent senescence with antitumor, cosmetic and immunity-enhancing effects.

Antioxidant activities of polysaccharide can be affected by chemical components, molecular mass, structure, conformation and even the drying methods. Drying has become a widely used method for the preparation of polysaccharides. Various techniques have been used in the drying process of polysaccharides. Traditional Hot air Drying (HD) is the most commonly used drying method in the food industry. However, significant quality changes of the dried product may occur during HD. Vacuum Drying (VD) is ideal for materials that can be damaged or changed by exposure to high temperatures and oxygen. Vacuum removes moisture while preventing oxidation that may occur when certain materials are combined with air (Fan et al., 2012). However, drying may lead to changes in the structure of polysaccharides, thereby affecting their biological activity. How to choose a suitable drying method for polysaccharides without affecting the biological activity of polysaccharides is particularly important?

Currently, there are few reports about the effects of different drying technologies on the properties of AMP. Therefore, the purpose of this study is to explore the effects of different drying methods on the composition and antioxidant activity of AMP. On the premise of maintaining high antioxidant activity of AMP, the present study aims to find a suitable drying method for AMP and lay the foundation for the industrial application of AMP. 


\section{Materials and Methods}

\section{Materials and Equipment}

Velvet Antler mushroom was from Shanghai Detian Agricultural Products Co., Ltd. Potassium ferricyanide $\left(\mathrm{K}_{3}\left[\mathrm{Fe}(\mathrm{CN})_{6}\right]\right)$ was from Shanghai Hongrui Chemical Industry Co., Ltd. DPPH was from Shanghai Hualan Chemical Technology Co., Ltd. Hydrochloric acid, anhydrous ethanol, methanol and phenol were of analytical grade from Jiangsu Qiangsheng Chemical Co., Ltd. China.

An SL68-GJ-3 sealed sample grinder was from Zhengzhou Zhongcheng environmental protection equipment Co., Ltd. HWS-26 constant temperature water bath was from Jiangsu Jiamei Instrument Manufacturing Co., Ltd. H1750R high-speed freezing centrifuge was from Hunan Hengnuo Instrument Equipment Co., Ltd. PGC-753E precision balance was from Adam Equipment. UV-1100 visible spectrophotometer was from Jinan Siquan Medical Equipment Co., Ltd. UV1800PC ultraviolet spectrophotometer was from Tsushima Instruments, Japan. RE-2000B rotary evaporator was from Lanfan Technology Co., Ltd. SHZ-D circulating water multi-purpose vacuum pump was from Zhengzhou Zituo Instrument Equipment Co., Ltd. FDGJ-5C vacuum freeze dryer was from Changzhou Panfeng Drying Equipment Co., Ltd. 101-0A digital display electric constant temperature blast drying oven was from Jinan Zhuolong Biotechnology Co., Ltd. NicoletIS10 Fourier infrared spectrometer was from Tianjin Energy Spectrum Technology Co., Ltd. LC2010 liquid chromatograph was from Shenzhen Huadelong Technology Co., Ltd. GC2014C Shimadzu gas chromatograph was from Shimadzu Corporation, Japan.

\section{Preparation of AMP}

The fresh Antler mushroom samples were rinsed with distilled water, drained and dried in a vacuum oven for 12 $\mathrm{h}$ at $60^{\circ} \mathrm{C}$. Then, the samples were mechanically crushed through 90 mesh screens and washed two times with 95\% ethanol to remove some pigments, oligosaccharides and other small molecular substances. The solvents were filtered and dried at $60^{\circ} \mathrm{C}$ for $24 \mathrm{~h}$ to obtain the Antler mushroom powder. The sample $(100 \mathrm{~g})$ was placed inside a tapered bottle and then $3000 \mathrm{~mL}$ distilled water was added. The mixture was extracted in a water bath at $75^{\circ} \mathrm{C}$ for $6 \mathrm{~h}$ and centrifuged at $3500 \mathrm{r} / \mathrm{min}$ for $30 \mathrm{~min}$ to obtain the extract. Then the extract was concentrated under reduced pressure to obtain the concentrate. The concentrate was deproteinized by Sevage method five times. The concentrate was purified by a polyamide adsorption column and eluted with distilled water to obtain the polysaccharide extract. Polyamide is a type of polymer compound formed by the polymerization of amide groups. Polyamide molecules are rich in amide groups, which can form hydrogen bonds with phenols, quinones and nitro compounds to be adsorbed. Therefore, the use of polyamide as a chromatographic column filler can separate and refine certain types of substances with a certain polarity range. After the polysaccharide extract was further dialyzed for $24 \mathrm{~h}, 95 \%$ ethanol (the volume was 4 times of dialysate) was added to precipitate at $2{ }^{\circ} \mathrm{C}$ for $12 \mathrm{~h}$. The precipitate (AMP) was obtained by centrifugation $(3500 \mathrm{r} / \mathrm{min})$.

\section{Drying of $A M P$}

AMP was dried by hot air drying, vacuum drying and freeze drying to obtain hot air-dried polysaccharides (AMP$\mathrm{H}$ ), vacuum-dried polysaccharides (AMP-V) and freezedried polysaccharides (AMP-F). According to our preliminary optimization experiments, the drying parameters of the three drying methods were determined as follows: hot air-drying, $70^{\circ} \mathrm{C}$ with a wind speed of $2.0 \mathrm{~m} / \mathrm{s}$ for $16 \mathrm{~h}$; vacuum-drying, $60^{\circ} \mathrm{C}$ with a vacuum degree of $0.07 \mathrm{MPa}$ for $12 \mathrm{~h}$; and freeze drying conditions, $-50^{\circ} \mathrm{C}$ with a pressure of $0.06 \mathrm{MPa}$ for $48 \mathrm{~h}$. All three AMP were brownish-yellow powders with a water content of less than or equal to $5 \%$.

\section{Analysis of AMP Composition}

Using glucose as the reference substance, the content of neutral sugar was determined by phenol-sulfuric acid method (Rasouli et al., 2014). Using galacturonic acid as the standard, Glycuronate content was determined by method of m-hydroxydiphenyl (Blumenkrantz and Gasboe, 1973). Using bovine serum albumin as the standard, the Folin-phenol method was used to determine protein (Lowry et al., 1951).

\section{Analysis of the Monosaccharide Composition of AMP}

The monosaccharide composition of AMP was analyzed using the derivatization of glyconitrile acetate in combination with the GC-MS (Grace et al., 2013). First, $10 \mathrm{mg}$ of AMP was weighed in a conical flask and added into $2 \mathrm{~mL}$ trifluoroacetic acid $(2 \mathrm{~mol} / \mathrm{L})$. The mixture was heated in a water bath at $100^{\circ} \mathrm{C}$ until fully hydrolyzed. Then $10 \mathrm{mg}$ sodium borohydride and an appropriate amount of inositol were mixed. The mixture was stored at room temperature for $8 \mathrm{~h}$. Acetic acid was used to remove excess sodium borohydride and added into $0.5 \mathrm{~mL}$ acetic anhydride and $0.5 \mathrm{~mL}$ pyridine after decompression drying. When shaking was completed, the acetylation reaction was carried out in a constant temperature water bath at $90^{\circ} \mathrm{C}$ for $6 \mathrm{~h}$ and the acetic anhydride and pyridine were dried under reduced pressure. The remaining products were dissolved in $\mathrm{CHCl}_{3}$ and finally analyzed using GC-MS over a $0.22 \mu \mathrm{m}$ microporous membrane. $0.25 \mu \mathrm{m} \times 0.25 \mathrm{~mm} \times 30 \mathrm{~m}$ ) was used. Gas carrier was $\mathrm{H}_{2}$. Flow rate was $1.5 \mathrm{~mL} / \mathrm{min}$. Injection temperature was $280^{\circ} \mathrm{C}$ and interface temperature was $230^{\circ} \mathrm{C}$. The 
temperature programmed was as follows: Starting temperature was $120^{\circ} \mathrm{C}$ for $3 \mathrm{~min}$ and then $210^{\circ} \mathrm{C}$ for 4 min.

\section{Fourier Transform Infrared (FTIR) Spectroscopy Analysis of $A M P$}

The infrared spectrum of AMP was analyzed by $\mathrm{KBr}$ pressing method. First, potassium bromide was backed at $110^{\circ} \mathrm{C}$ in an oven for $2 \mathrm{~h}$ and dried. Different drying methods obtained AMP with the rate of KBr: Sample $=100: 1$ in a dry agate bowl and mixed and pressed into transparent sheets. Scanning was performed using an FTIR spectrometer ranging from 4000 to $400 \mathrm{~cm}^{-1}$ at a resolution ratio of $4 \mathrm{~cm}^{-1}$ (Wang et al., 2017).

\section{Observation of AMP Morphology}

First, the sample powder of AMP-F was glued to the copper sample table, the floating sample was blown off and the surface was sprayed with gold. Finally, the morphology of the sample was scanned and observed by electron microscopy.

\section{Determination of Antioxidant Activity}

\section{Determination of DPPH Scavenging Activity}

The DPPH scavenging activity was determined by adopting the reported methods (Giri et al., 2011). $1 \mathrm{~mL}$ AMP-H, AMP-V and AMP-F (0.5, 1.0, 2.0, 3.0 and 4.0 $\mathrm{mg} / \mathrm{mL}$ ) were placed in a test tube and added into $2 \mathrm{~mL}$ DPPH-ethanol solution $(0.1 \mathrm{mmol} / \mathrm{L})$. The solution was shaken well and stored away from light for $30 \mathrm{~min}$. The absorbance was determined at $517 \mathrm{~nm}$ using a spectrophotometer. The sample determination was performed three times. The mean value was recorded. DPPH radical scavenging rate was calculated according to the following formula:

$$
\text { DPPH radical scavenging rate }(\%)=\left[1-\left(A_{1}-A_{2}\right) / A_{0}\right] \times 100
$$

where $\mathrm{A}_{1}$ is the absorbance with the presence of DPPH and tested polysaccharides. $A_{0}$ is the absorbance of the blank group without polysaccharide sample. $A_{2}$ is the absorbance of the control group with the presence of tested polysaccharides but without DPPH.

\section{Determination of Superoxide Anion Scavenging Activity}

The superoxide anion radical scavenging effect was determined using the reported method (Jiang et al., 2010) with slight modifications. $4.5 \mathrm{~mL}$ Tris- $\mathrm{HCl}$ buffer solution (50 mmol/L, pH 8.2), $1 \mathrm{~mL}$ AMP-H, AMP-V and AMPF solutions $(0.5,1.0,2.0,3.0$ and $4.0 \mathrm{mg} / \mathrm{mL})$ and $0.4 \mathrm{~mL}$ pyrogallic acid $(3.0 \mathrm{mmol} / \mathrm{L})$ were mixed and stood at $25^{\circ} \mathrm{C}$ for $20 \mathrm{~min}$. Then the mixed solutions were heated for $5 \mathrm{~min}$ at $25^{\circ} \mathrm{C}$. Thereafter, $0.5 \mathrm{~mL}$ thick hydrochloric acid was added to terminate the reaction. Absorbance was determined at $299 \mathrm{~nm}$ and recorded as $A_{1}$. The blank control group was replaced by the polysaccharide sample solution with Tris-HCl buffer. The sample determination was repeated three times. The mean value was recorded. Superoxide anion radical scavenging rate was calculated according to the following formula:

$$
\begin{aligned}
& \text { Superoxide anion scavenging rate }(\%) \\
& =\left(A_{0}-A_{1}\right) / A_{0} \times 100
\end{aligned}
$$

where $A_{0}$ is the absorbance value of Tris- $\mathrm{HCl}$ buffer instead of polysaccharide sample. $A_{1}$ is the absorbance value of polysaccharide sample at various concentrations.

\section{Determination of Hydroxyl Radical Scavenging Activity}

$1 \mathrm{~mL}$ AMP-H, AMP-V and AMP-F solutions $(0.5,1.0$, 2.0, 3.0 and $4.0 \mathrm{mg} / \mathrm{mL}), 1 \mathrm{~mL} \mathrm{FeSO}_{4}$ solution $(9.0 \mathrm{mmol} / \mathrm{L})$ and $1 \mathrm{~mL}$ salicylic acid ethanol solution $(9.0 \mathrm{mmol} / \mathrm{L})$ were mixed. $1.0 \mathrm{~mL} \mathrm{H} \mathrm{H}_{2}(8.8 \mathrm{mmol} / \mathrm{L})$ were added into the mixture to start the reaction. The mixed reaction solution was incubated at $37^{\circ} \mathrm{C}$ for $30 \mathrm{~min}$. Absorbance at $562 \mathrm{~nm}$ was determined as $A_{1}$. Distilled water instead of polysaccharide sample solution was recorded at $562 \mathrm{~nm}$, which was recorded as $A_{0}$. In the blank control group, distilled water was used instead of salicylic acid ethanol solution. The absorbance at $562 \mathrm{~nm}$ was measured and recorded as $A_{2}$. The measurement was performed three times. The hydroxyl radical scavenging rate was calculated as follows:

Hydroxyl radical scavenging rate $(\%)=\left[1-\left(A_{1}-A_{2}\right) / \mathrm{A}_{0}\right] \times 100$

where $A_{1}$ is the absorbance of polysaccharide sample. $A_{2}$ is the absorbance without salicylic acid-ethanol solution. $A_{0}$ is the absorbance of the control (distilled water instead of the polysaccharides).

\section{Results and Discussion}

\section{Chemical Constituents of AMP}

Extracted polysaccharides from natural products often have neutral sugars and glyoxylic acid. Covalent bonds with proteins form proteoglycans with biological functions (Ma et al., 2013). Neutral sugars, glyoxylic acid and proteins were measured in AMP-H, AMP-V and AMP-F as shown in Table 1. Acidic polysaccharides, referred to as polysaccharides containing acidic groups, mainly include glyoxylic acid, sulfuric acid group and phosphoric acid group. Their chemical structures may also contain neutral sugar. Therefore, the three polysaccharides are all acidic polysaccharides containing a small amount of protein (Yan et al., 2019). The content of neutral sugar and 
protein in the three polysaccharides was low. The content of uronic acid was 4.09, 4.91 and 4.84\% in AMP-H, AM P-V and AMP-F, respectively. The content of uronic acid in AMP-V and AMP-F was significantly higher than that in AMP-H $(p<0.05)$. The possible reason is that structure of the uronic acid in AMP-H is destroyed under dry, oxygen and high temperature conditions (Chen and Huang, 2019). In this experiment, the uronic acid content of AMP-H, AMP-V and AMP-F was less than 5\%. The reason may be that neutral sugars and uronic acid interacted, not both independent. They may exist in a sugar chain and cannot be separated using existed methods. On the other hand, the content of neutral sugar and uronic acid is determined by different methods, which will interfere with each other.

\section{Monosaccharide Composition of AMP}

AMP monosaccharide composition was analyzed by GC-MS technique. Table 2 showed that the monosaccharide compositions of the three AMP were galactose, ribose, mannose, fucose, rhamnose, glucose and xylose, but its molar ratio had changed. The monosaccharide molar ratios of AMP-H, AMP-V and AMP-F were 1:13.63:50.62:34.38:284.08:80.36,1:5.24:69.09:45.26:300.3 $1: 70.41$ and $1: 6.82: 73.18: 25.71: 332.05: 63.75$, respectively. The results showed that the drying method generally did not change the monosaccharide species of polysaccharides but affected their composition ratios, which may be due to the change in monosaccharide conformation during drying (Wang et al., 2013). Besides, different drying methods can directly affect the molecular weight and spatial composition of polysaccharides (Wu, 2015).

\section{Fourier Transform Infrared Spectroscopy Analysis of $A M P$}

According to the positions and characteristics of the characteristic peaks in the infrared spectrum, the structural characteristics of polysaccharides can be inferred. Therefore, the basic method which can qualitatively identify the structure of polysaccharides is mainly infrared spectroscopy. Figure 1 showed that the infrared spectra of AMP-H, AMP$\mathrm{V}$ and AMP-F was the same. In the range of $4000-400 \mathrm{~cm}^{-1}$, AMP-H, AMP-V and AMP-F had the general characteristics of polysaccharides. AMP-H, AMP-V and AMP-F showed strong peaks at 3400, 2900, 1600 and $1400 \mathrm{~cm}^{-1}$. The strong absorption peak near $3400 \mathrm{~cm}^{-1}$ is mainly attributed to $\mathrm{O}-\mathrm{H}$ and N-H stretching vibrations. The absorption peak at 2900 $\mathrm{cm}^{-1}$ is attributed to the stretching vibration of the $\mathrm{C}-\mathrm{H}$ bond. The absorption peaks at 1600 and $1400 \mathrm{~cm}^{-1}$ are attributable to the stretching vibration of the $\mathrm{C}-\mathrm{H}$ bond and the deformation characteristics of $=\mathrm{CH}_{2}$. The absorption peaks around 1600 and $1400 \mathrm{~cm}^{-1}$ indicated the presence of carboxylic acids in polysaccharides (Liu et al., 2017). Therefore, AMP-H, AMP-V and AMP-F were acid polysaccharides. The absorption peak at $600 \mathrm{~cm}-1$ was the skeleton symmetric stretching vibration absorption peak of pyranose (Černá et al., 2003). Therefore, AMP is a kind of pyranose.

\section{Electron Microscope Analysis}

Figure 2 showed the AMP morphology under a scanning electron microscope. At $2500 \times$ magnification, the polysaccharide samples were mostly reticular, indicating that the polysaccharide had a high molecular weight, long-chain or branched structure and ability to cross adhere between molecules easily. After 5000 times of exposure, the surface of the polysaccharide samples showed a smooth and flat sheet structure, indicating the interaction between polysaccharides and their ability to form tight structures easily (Shang et al., 2013).

\section{Antioxidant Effect of AMP in Vitro}

\section{Scavenging Effect of AMP-H, AMP-V and AMP-F on DPPH Free Radicals}

The DPPH assay was used to assess antioxidant activity of extracted polysaccharides. DPPH is a relatively stable free radical and can accept an electron or hydrogen radical to become a stable diamagnetic molecule, which has been widely accepted as a tool for estimating the freeradical scavenging activities of antioxidants (Ke et al., 2015). The scavenging effects of polysaccharides named GLP-F, GLP-V and GLP-H on the DPPH radical were in a concentration-dependent fashion (Fan et al., 2012).

Figure 3 showed that the AMP obtained by hot air drying, freeze drying and vacuum drying have a good ability of scavenging DPPH free radicals. The scavenging effect was positively correlated with the mass concentration. As the mass concentration of $t$ AMP-H, AMP-V and AMP-F increased, the scavenging effect also increased. The scavenging effect of AMP-F, AMP-V and AMP-H on DPPH free radicals weakened in turn. When the polysaccharide concentration reached $4.0 \mathrm{mg} / \mathrm{mL}$, DPPH free radical scavenging rate of AMP-H, AMP-V and AMP-F was 58.9, 65.0 and 89.1\%, respectively. The DPPH radical scavenging abilities were influenced by the different drying method because of the changes of the physicochemical properties of polysaccharide samples (Ma et al., 2013).

\section{Scavenging Effect of AMP-H, AMP-V and AMP-F on Superoxide Anion Radicals}

Superoxide anion free radicals can accelerate the aging process from the skin to the internal organs (Lagercrantz, 1999). Accordingly, it can induce many diseases by damaging important biological macromolecules (Sun et al., 2009). Figure 4 showed that AMP obtained by hot air drying, freeze drying and vacuum drying can effectively scavenge superoxide anion free radicals. The scavenging effect of the three AMP polysaccharides on superoxide anion radical increased when concentration increased. 
AMP-F had the strongest scavenging ability, whereas AMP-H had the weakest. When the polysaccharide concentration reached $4.0 \mathrm{mg} / \mathrm{mL}$, the scavenging rate of AMP-H, AMP-V and AMP-F on superoxide anion radical was $59.5,62.2$ and $88.3 \%$, respectively.

\section{Scavenging Effect of AMP-H, AMP-Vand AMP-F on} Hydroxyl Radicals

Hydroxyl radical is considered to be a strong oxidant, which can interact with most biological macromolecules and induce serious damage to the adjacent biomolecules (Fan et al., 2012). Therefore, scavenging hydroxyl free radicals is important for the antioxidant defense in the cell or food system.

Figure 5 showed that AMP dried by hot air drying, freeze drying and vacuum drying can scavenge hydroxyl radical as well. With the increase of the concentration of AMP-H, AMP-V and AMP-F, the scavenging effect of hydroxyl radicals increased within a certain concentration range. When the concentration was $4 \mathrm{mg} / \mathrm{mL}$, the scavenging effect of AMP-H, AMP-V and AMP-F on hydroxyl free radicals is the highest, respectively. Among them, the scavenging effect of AMP-F on hydroxyl free radicals is higher than that of AMP-H and AMP-V.

Table 1: Effects of different drying methods on the chemical composition of AMP

\begin{tabular}{llll}
\hline Sample & AMP-H & AMP-V & AMP-F \\
\hline Neutral sugar (\%) & $85.20 \pm 1.75 \mathrm{a}$ & $85.16 \pm 1.12 \mathrm{a}$ & $86.23 \pm 1.62 \mathrm{a}$ \\
Protein (\%) & $2.26 \pm 0.12 \mathrm{a}$ & $2.38 \pm 0.17 \mathrm{a}$ & $2.34 \pm 0.10 \mathrm{a}$ \\
Uronic acid (\%) & $4.09 \pm 0.25 \mathrm{~b}$ & $4.91 \pm 0.31 \mathrm{a}$ & $4.84 \pm 0.27 \mathrm{a}$ \\
\hline
\end{tabular}

Note: The data in the table are the average \pm standard deviation of the data repeated 3 times. The data corresponding to the same peer with different lowercase letters is significant $(p<0.05)$

Table 2: Effects of different drying methods on AMP monosaccharide composition

\begin{tabular}{llll}
\hline Sample & AMP-H & AMP-V & AMP-F \\
\hline Xylose (\%) & $41.69 \pm 0.19 \mathrm{~b}$ & $43.00 \pm 0.22 \mathrm{~b}$ & $49.27 \pm 0.91 \mathrm{a}$ \\
Glucose (\%) & $28.84 \pm 0.32 \mathrm{a}$ & $25.67 \pm 0.29 \mathrm{~b}$ & $23.60 \pm 0.50 \mathrm{c}$ \\
Galactose (\%) & $1.21 \pm 0.01 \mathrm{c}$ & $2.73 \pm 0.04 \mathrm{a}$ & $2.65 \pm 0.13 \mathrm{~b}$ \\
Mannose (\%) & $3.13 \pm 0.57 \mathrm{a}$ & $2.42 \pm 0.35 \mathrm{~b}$ & $2.41 \pm 0.09 \mathrm{~b}$ \\
Rhamnose (\%) & $16.89 \pm 0.88 \mathrm{~b}$ & $20.02 \pm 0.34 \mathrm{a}$ & $10.99 \pm 0.18 \mathrm{c}$ \\
Ribose (\%) & $0.21 \pm 0.0004 \mathrm{c}$ & $0.45 \pm 0.0038 \mathrm{~b}$ & $0.90 \pm 0.0030 \mathrm{a}$ \\
Fucose (\%) & $11.31 \pm 0.63 \mathrm{c}$ & $17.24 \pm 0.18 \mathrm{~b}$ & $23.07 \pm 0.45 \mathrm{a}$ \\
\hline
\end{tabular}

Note: The data in the table are the average \pm standard deviation of the data repeated 3 times. The data corresponding to the same peer with different lowercase letters is significant $(p<0.05)$

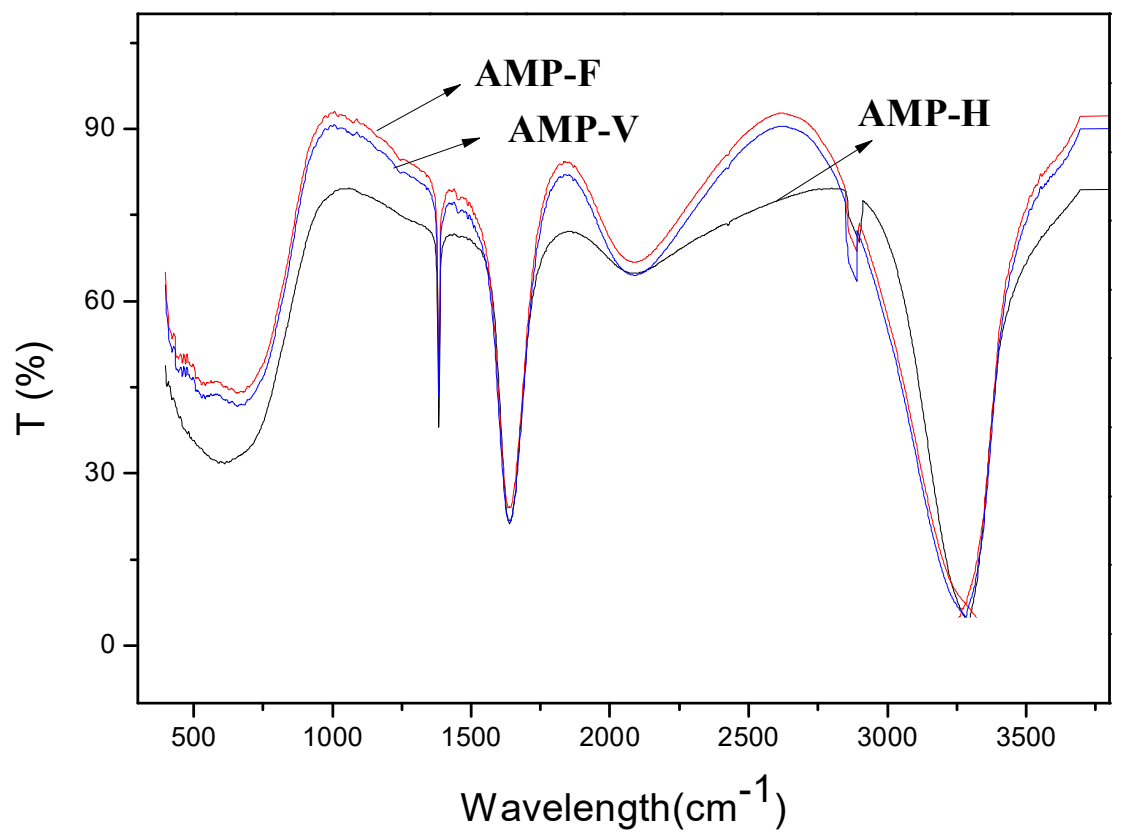

Fig. 1: Infrared spectra of AMP dried by different methods 


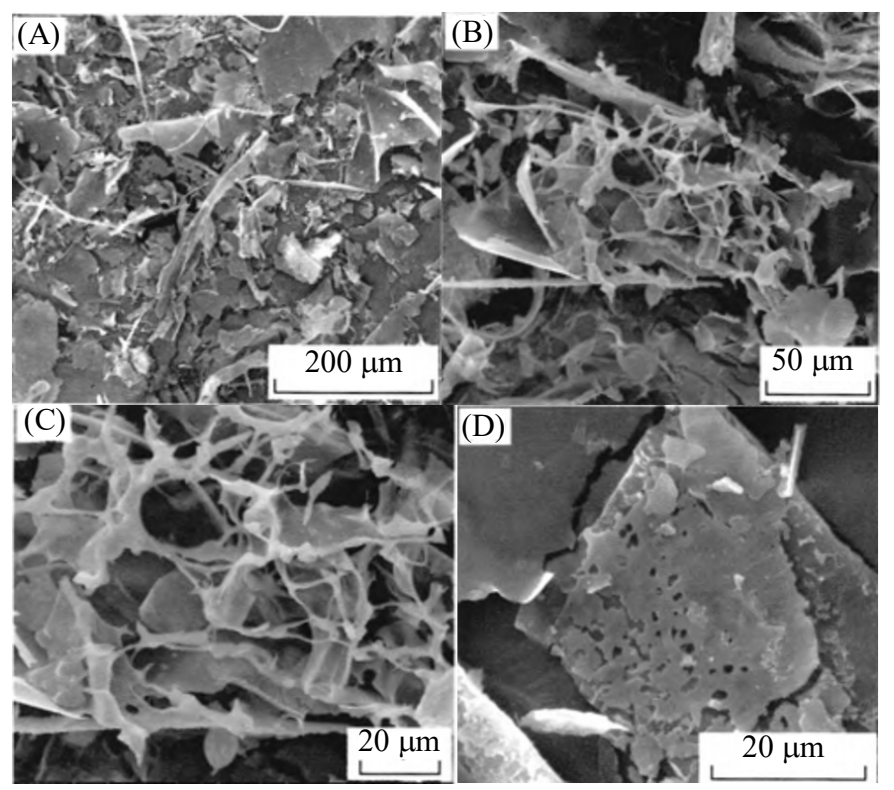

Fig. 2: Observation of AMP under electron microscope with different magnification at 500(A), 1300(B), 2500(C) and 5000(D)

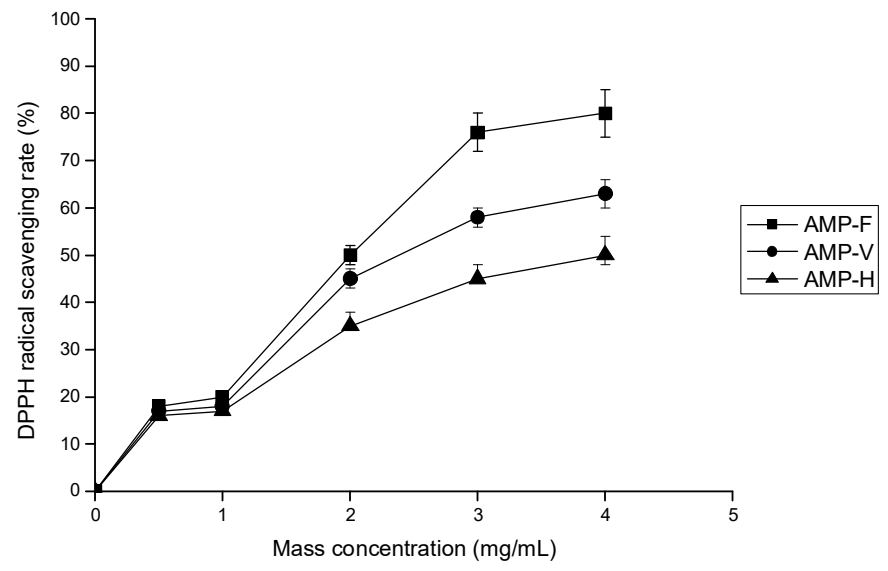

Fig. 3: Scavenging effect of AMP dried by different methods on DPPH free radicals

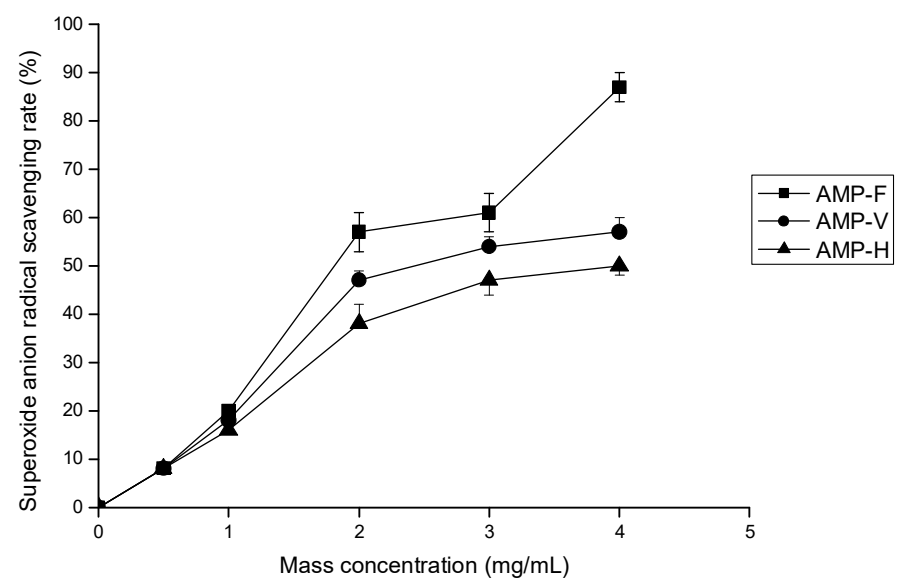

Fig. 4: Scavenging effect of AMP dried by different methods on superoxide anion free radicals 


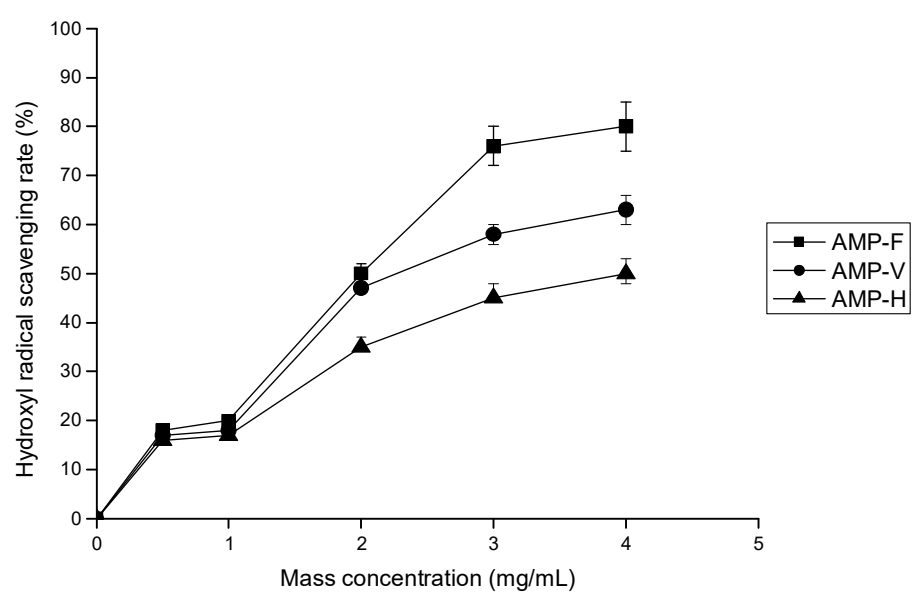

Fig. 5: Scavenging effect of AMP dried by different methods on hydroxyl radicals

\section{Conclusion}

In this study, hot air drying, freeze-drying and vacuum-drying were used to dry AMP to obtain AMP-H, AMP-V and AMP-F, respectively. The chemical composition and antioxidant capacity of AMP-H, AMP$\mathrm{V}$ and AMP-F were investigated. AMP-H, AMP-V and AMP-F were all acidic polysaccharides containing a small amount of protein. The monosaccharide species of AMP-H, AMP-V and AMP-F were unchanged, whereas their monosaccharide compositions were different. The content of uronic acid in AMP-H was lower significantly than that in AMP-V and AMP-F $(\mathrm{p}<0.05)$. The infrared spectrum analysis showed that the chemical structure of AMP-H, AMP-V and AMP-F was basically the same. The antioxidant activity in vitro showed that drying mode had a significant effect on the antioxidant ability of AMP. Compared with AMP-V and AMP-H, AMP-F had the strongest antioxidant activity. Therefore, freeze drying is the best drying method for preparing AMP. This work provides a theoretical basis for the development and utilization of AMP.

\section{Acknowledgement}

This research was supported by Suzhou Science and Technology Plan Project (SNG2020064).

\section{Author's Contributions}

Bing $\mathrm{Xu}$ and Yi Dai: Participated in all experiments and article writing.

Shiyu Xu, Jianjun Liu and Botao Hu: Participated in drying of AMP and data processing.

Xiao Ding and Xinyi Wu: Participated in antioxidant activities of AMP-H, AMP-V and AMP-F.

Yiyong Chen: Participated in project design and experimental guidance of this manuscript.

\section{Ethics}

This article is original and contains unpublished material. The corresponding author confirms that all of the other authors have read and approved the manuscript and no ethical issues involved.

\section{References}

Arana-Gabriel, Y., Burrola-Aguilar, C., Garibay-Orijel, R., Matías-Ferrer, N., Franco-Maass, S., \& Mata, G. (2018). Genetic characterization, evaluation of growth and production of biomass of strains from wild edible mushrooms of Lyophyllum of Central Mexico. brazilian journal of microbiology, 49, 632640. https://doi.org/10.1016/j.bjm.2017.12.002

Blumenkrantz, N., \& Asboe-Hansen, G. (1973). New method for quantitative determination of uronic acids. Analytical biochemistry, 54(2), 484-489. https://doi.org/10.1016/0003-2697(73)90377-1

Černá, M., Barros, A. S., Nunes, A., Rocha, S. M., Delgadillo, I., Čopíková, J., \& Coimbra, M. A. (2003). Use of FT-IR spectroscopy as a tool for the analysis of polysaccharide food additives. Carbohydrate Polymers, 51(4), 383-389. https://doi.org/10.1016/S0144-8617(02)00259-X

Chen, F., \& Huang, G. (2019). Antioxidant activity of polysaccharides from different sources of ginseng. International journal of biological macromolecules, 125, 906-908. https://doi.org/10.1016/j.ijbiomac.2018.12.134

Fan, L., Li, J., Deng, K., \& Ai, L. (2012). Effects of drying methods on the antioxidant activities of polysaccharides extracted from Ganoderma lucidum. Carbohydrate Polymers, 87(2), 1849-1854. https://doi.org/10.1016/j.carbpol.2011.10.018 
Giri, A., Osako, K., Okamoto, A., Okazaki, E., \& Ohshima, T. (2011). Antioxidative properties of aqueous and aroma extracts of squid miso prepared with Aspergillus oryzae-inoculated koji. Food Research International, 44(1), 317-325. https://doi.org/10.1016/j.foodres.2010.10.013

Grace, O. M., Dzajic, A., Jäger, A. K., Nyberg, N. T., Önder, A., \& Rønsted, N. (2013). Monosaccharide analysis of succulent leaf tissue in Aloe. Phytochemistry, 93, 79-87. https://doi.org/10.1016/j.phytochem.2013.03.015

Jiang, B., Zhang, H., Liu, C., Wang, Y., \& Fan, S. (2010). Extraction of water-soluble polysaccharide and the antioxidant activity from Ginkgo biloba leaves. Medicinal Chemistry Research, 19(3), 262-270. https://doi.org/10.1007/s00044-009-9189-5

Ke, C. L., Wang, D., Zeng, W. G., Yao, X. Y., Xu, H., \& Zeng, X. X. (2015). Antioxidant and immunostimulatory activities In Vitro of Polysaccharides from Pomegrante Peels. Journal of the Chemical Society of Pakistan, 37(1).

Lagercrantz, C. (1999). Formation of the paramagnetic complex $[\mathrm{Cr}(\mathrm{OH}) 5 \mathrm{O} 2] 5-$ in the reaction between chromium (VI) oxide and hydrogen peroxide or superoxide anion radicals studied by EPR spectroscopy. Free Radical Biology and Medicine, 26(9-10), 1134-1137. https://doi.org/10.1016/S08915849(98)00294-9

Lee, D. J., Lee, W. J., Kuen, M. S., Jang, J. W., Lee, W. J., Her, J. R., \& Lee, B. E. (2011). Studies on the mutant introduction and new practical artificial cultivation of Lyophyllum decastes. Journal of Mushroom, 9(2), 63-68.

https://www.koreascience.or.kr/article/JAKO201114 364645151.page

Liu, D., Sun, Q., Xu, J., Li, N., Lin, J., Chen, S., \& Li, F. (2017). Purification, characterization and bioactivities of a polysaccharide from mycelial fermentation of Bjerkandera fumosa. Carbohydrate Polymers, 167, 115-122. https://doi.org/10.1016/j.carbpol.2017.03.029

Lowry, O. H., Rosebrough, N. J., Farr, A. L., \& Randall, R. J. (1951). Protein measurement with the Folin phenol reagent. Journal of Biological Chemistry, 193, 265-275. https://doi.org/10.1016/S0021-9258(19)52451-6

Ma, L., Chen, H., Zhu, W., \& Wang, Z. (2013). Effect of different drying methods on physicochemical properties and antioxidant activities of polysaccharides extracted from mushroom Inonotus obliquus. Food Research International, 50(2), 633-640. https://doi.org/10.1016/j.foodres.2011.05.005
Rasouli, M., Ostovar-Ravari, A., \& Shokri-Afra, H. (2014). Characterization and improvement of phenol-sulfuric acid microassay for glucose-based glycogen. Eurgy Revised Medicine Pharmacology Science, 2020-2024. http://www.europeanreview.org/wp/wpcontent/uploads/2020-2024.pdf

Shang, S., Zhu, L., \& Fan, J. (2013). Intermolecular interactions between natural polysaccharides and silk fibroin protein. Carbohydrate Polymers, 93(2), 561573. https://doi.org/10.1016/j.carbpol.2012.12.038

Sun, H. J., Jiang, S., Zi, M., \& Qi, D. (2009). Purification, chemical composition and in vitro antioxidant activity of two protein-bound polysaccharides from rapeseed meal. Food Science and Biotechnology, 18(6), 1386-1391. https://www.koreascience.or.kr/article/JAKO2009 10348028287.page

Wang, X. J., Shan, X. D., Hu, M. H., Cai, C., Zhao, X. L., Fan, L. D., Lv, Y. J., Hao, J. J., Li, G. Y., Yu, G. L. (2017). Structure characterization of Cyclocarya paliurus leaf polysaccharide and its inhibitory activity on $\alpha$-glucosidase. Chinese Traditional and Herbal Drugs, 48(8), 1524-1528(in Chinese).

Wang, Y., Liu, Y., Huo, J., Zhao, T., Ren, J., \& Wei, X. (2013). Effect of different drying methods on chemical composition and bioactivity of tea polysaccharides. International Journal of Biological Macromolecules, $\quad 62, \quad 714-719$. https://doi.org/10.1016/j.ijbiomac.2013.10.006

$\mathrm{Wu}, \mathrm{Z}$. (2015). Effect of different drying methods on chemical composition and bioactivity of finger citron polysaccharides. International Journal of Biological Macromolecules, 76, 218-223. https://doi.org/10.1016/j.ijbiomac.2015.02.043

Yan, J., Zhu, L., Qu, Y., Qu, X., Mu, M., Zhang, M., ... \& Sun, L. (2019). Analyses of active antioxidant polysaccharides from four edible mushrooms. International Journal of Biological Macromolecules, 123, 945-956. https://doi.org/10.1016/j.ijbiomac.2018.11.079

Zheng, H. L., Bau, T., Bao, H. Y., \& Lian, J. W. (2013). Chemical constituents from Lyophyllum decastes. Zhongguo Zhong yao za zhi= Zhongguo zhongyao zazhi $=$ China journal of Chinese materia medica, 38(24), 4335-4339. https://europepmc.org/article/med/24791541p 